\title{
Comparative evaluation of the effect of different growth media on in vitro sensitivity to azithromycin in multi-drug resistant Pseudomonas aeruginosa isolated from cystic fibrosis patients
}

\author{
Michael Sörensen ${ }^{1,2^{*}}$, Bakhodur Khakimov ${ }^{1}$, Dennis Nurjadi ${ }^{1}$, Sébastien Boutin ${ }^{1}$, Buqing Yi ${ }^{3}$,
} Alexander H. Dalpke ${ }^{1,3^{*}}$ (1) and Tatjana Eigenbrod ${ }^{1,4^{*}}$

\begin{abstract}
Long-term treatment with azithromycin is a therapeutic option in Cystic Fibrosis (CF) patients chronically infected with $P$. aeruginosa. It was recently shown that azithromycin has direct antimicrobial activity when $P$. aeruginosa isolates are tested in Roswell Park Memorial Institute medium supplemented with fetal calf serum (RPMI 1640/FCS) by broth microdilution. We now investigated whether (i) azithromycin might also be active against multidrug resistant (MDR) $P$. aeruginosa isolated from CF patients and (ii) how in vitro sensitivity assays perform in synthetic cystic fibrosis sputum medium (SCFM), a medium that mimics the particular CF airway environment.In 17 (59\%) out of 29 MDR P. aeruginosa CF isolates MICs for azithromycin ranged between 0.25 and $8 \mu \mathrm{g} / \mathrm{ml}$ and 12 isolates (41\%) showed a MIC $\geq 512 \mu \mathrm{g} /$ $\mathrm{ml}$ when measured in RPMI/FCS. In contrast, MICs were $\geq 256 \mu \mathrm{g} / \mathrm{ml}$ for all P. aeruginosa MDR isolates when tested in either SCFM or in conventional cation-adjusted Mueller Hinton Broth. High MIC values observed in CF adapted medium SCFM for both PAO1 and MDR P. aeruginosa CF isolates, as opposed to findings in RPMI, argue against routine azithromycin MIC testing of CF isolates.
\end{abstract}

Keywords: Cystic fibrosis, Pseudomonas aeruginosa, Multidrug resistance, Broth microdilution, Azithromycin, SCFM

\section{Background}

Cystic fibrosis (CF) is a chronic disorder caused by autosomal-recessive mutations in the cystic fibrosis transmembrane conductance regulator (CFTR) gene. Airway infections with Pseudomonas aeruginosa (P. aeruginosa)

\footnotetext{
*Correspondence: m.soerensen@labor-enders.de; alexander.dalpke@ukdd.de; tatjana.eigenbrod@slk-kliniken.de

${ }^{1}$ Department of Infectious Diseases, Medical Microbiology and Hygiene, Heidelberg University Hospital, 69120 Heidelberg, Germany

${ }^{2}$ Laboratory Enders and Partners, 70193 Stuttgart, Germany

${ }^{3}$ Institute of Medical Microbiology and Hygiene, Medical Faculty,

Technische Universität Dresden, Fetscherstraße 74, 01307 Dresden, Germany

Full list of author information is available at the end of the article
}

are common in CF patients. They are associated with a decline in lung function, thereby contributing to increased mortality [1]. Current therapeutic strategies aim at eradicating initial or first infection with $P$. aeruginosa. When eradication fails, chronic infection can develop and then therapy tries to suppress $P$. aeruginosa load $[2,3]$.

Yet, antibiotic targeting of $P$. aeruginosa can be challenging as approximately $20 \%$ of $P$. aeruginosa positive patients have been reported by the North American CF registry to carry multidrug resistant (MDR) strains, as defined by resistance to all routinely tested antibiotics in two or more of the following classes: $\beta$-lactams, original author(s) and the source, provide a link to the Creative Commons licence, and indicate if changes were made. The images or other third party material in this article are included in the article's Creative Commons licence, unless indicated otherwise in a credit line to the material. If material is not included in the article's Creative Commons licence and your intended use is not permitted by statutory regulation or exceeds the permitted use, you will need to obtain permission directly from the copyright holder. To view a copy of this licence, visit http://creativecommons.org/licenses/by/4.0/. The Creative Commons Public Domain Dedication waiver (http://creativeco mmons.org/publicdomain/zero/1.0/) applies to the data made available in this article, unless otherwise stated in a credit line to the data. 
fluoroquinolones and aminoglycosides [4]. In these patients, inhaled antibiotics such as tobramycin, colistin and aztreonam have been suggested as therapy of choice due to the high concentrations that can be achieved upon local delivery [5].

Moreover, in patients chronically infected with $P$. aeruginosa, long-term treatment with macrolides, especially azithromycin, is an accepted therapeutic option and is progressively becoming standard of care [5-9]. The positive effect of azithromycin on clinically relevant end points, including increase in FEV1 (forced expiratory volume in $1 \mathrm{~s}$, a parameter used to assess lung function) and lower risk of pulmonary exacerbations [9], has primarily been attributed to anti-inflammatory and anti-virulence activities of azithromycin. Indeed, sub-inhibitory concentrations of azithromycin have been demonstrated to impair motility, quorum sensing and virulence factor expression including protease activity in $P$. aeruginosa [10].

Although traditionally $P$. aeruginosa is considered to be intrinsically resistant to macrolides, recent data indicate that macrolides may possess an in vitro antimicrobial activity against $P$. aeruginosa depending on the medium used for susceptibility testing by broth microdilution (BMD) $[11,12]$. Thus, minimal inhibitory concentrations (MICs) of azithromycin were significantly lower in Roswell Park Memorial Institute medium (RPMI 1640), a medium commonly used for culturing eukaryotic cells, in bronchoalveolar lavage fluid or in cation-adjusted Mueller Hinton Broth (CAMHB) supplemented with serum as compared to MICs measured conventionally in CA-MHB alone $[11,12]$. Therefore, it was suggested that MIC assessment of azithromycin in P. aeruginosa CF isolates using RPMI 1640 could be implemented as routine diagnostic measurement in microbiology laboratories [12].

However, it has not been studied whether azithromycin also exhibits antimicrobial activity against MDR $P$. aeruginosa, especially in the context of CF disease. Of note, previously used test media like RPMI1640/ FCS do not truly reflect the physiological airway environment observed in CF patients which might affect the interpretation of antibiotic susceptibility. In the present study, we therefore set out (i) to investigate the in vitro efficacy of azithromycin in MDR $P$. aeruginosa isolates derived from respiratory specimen of CF patients by (ii) using different media for BMD. Test media were CA-MHB, RPMI 1640 and synthetic cystic fibrosis sputum medium (SCFM), a medium mimicking the nutritional composition of CF sputum that was suggested to reflect physiological conditions [13].

\section{Methods}

Study population and routine microbiological analysis of samples

The study was done as a retrospective study on $P$. aeruginosa strains stored from CF patients who received in- or out-patient medical care at the University Hospital between January 2013 and December 2016. From those patients $P$. aeruginosa strains that were tested in the routine microbiology laboratory had been stored in skim milk at $-80^{\circ} \mathrm{C}$. The surveillance of multi-resistant organisms is performed in concordance to the German Infection Protection Act. The local ethics advisory board of the Heidelberg University Hospital was consulted prior to study begin for conformity with the current regulations (S-474/2018). Strain selection is described in the results section in detail. Identification at the species level of isolates cultured from respiratory samples was performed with MALDI-TOF (Bruker) and/or VITEK ${ }^{\circledR} 2$ (Biomerieux). Routine susceptibility testing was performed on the VITEK $^{\circledR} 2$ system for fast growing isolates using the VITEK ${ }^{\circledR} 2$ test card AST-N248 for gram-negative bacteria, while agar diffusion was used for slowly growing isolates in accordance with current German guidelines for microbiological laboratory standards [14]. Evaluation of colistin susceptibility was performed additionally within this study in cryopreserved isolates by BMD in concordance with current EUCAST (European Committee on Antimicrobial Susceptibility Testing) recommendations [15] using the commercially available Micronaut-S MIC strips (Merlin Diagnostics, Germany). All data for antimicrobial susceptibility testing were interpreted according to EUCAST clinical breakpoints.

\section{Determination of azithromycin MIC by BMD}

Azithromycin MICs were determined by BMD in 96-well microtiter plates in a concentration range of $0.125 \mu \mathrm{g} /$ $\mathrm{ml}$ to $1024 \mu \mathrm{g} / \mathrm{ml}$ according to current diagnostic standards [16, 17]. Briefly, P. aeruginosa clinical isolates or laboratory control strain PAO1 were grown overnight on Columbia Blood Agar plates at $36+/-1^{\circ} \mathrm{C}$ and inoculated into CA-MHB, RPMI 1640 with stable glutamine supplemented with $30 \%$ fetal calf serum (FCS) or synthetic cystic fibrosis sputum medium (SCFM) [13] at a final concentration of $5 \times 10^{5} \mathrm{CFU} / \mathrm{ml}$. Microtiter plates were incubated at $36+/-1{ }^{\circ} \mathrm{C}$ at ambient atmosphere, according to EUCAST guidelines and in line with Buyck et al. [11] MICs were routinely read at $20 \mathrm{~h}$. For some isolates, incubation was extended to $48 \mathrm{~h}$ due insufficient growth at $20 \mathrm{~h}$. MICs were read as the lowest concentration of azithromycin at which visible growth was inhibited. Two isolates failed to grow in SCFM and were therefore excluded from analysis. Reference strain PAO1 
has been described previously [18] and was obtained from the Leibniz Institute DSMZ (German Collection of Microorganisms and Cell Lines, \#22644).

\section{Determination of $P$. aeruginosa growth curves}

Bacterial growth curves were evaluated by using the Cell Growth Quantifier system (CGQ, Aquila Biolabs). CGQ is a technology for non-invasive real-time monitoring of biomass in shake flasks which is based on the measurement of the amount of light scattered towards a sensor as a function of the current biomass concentration inside the flask. To this end, $P$. aeruginosa was inoculated at a final concentration of $5 \times 10^{5} \mathrm{CFU} / \mathrm{ml}$ into the indicated culture media, transferred into Erlenmeyer conical flasks and shaken in the dark in $5 \% \mathrm{CO} 2$, endvolume $10 \mathrm{ml}$, at $36+/-1{ }^{\circ} \mathrm{C}, 200 \mathrm{rpm}$. Backscattered light was continuously measured by CGQ over $24 \mathrm{~h}$.

\section{S rRNA mutation detection}

PCR amplification and sequencing of domain $\mathrm{V}$ of $23 \mathrm{~S}$ rRNA genes of $P$. aeruginosa isolates, for which mutations have been described, were done as described previously [12] with forward primer: 5'-GGTGCCGGAAGG TTAATTGATG- $3^{\prime}$, and reverse primer: 5'-GCAGCC CCTCTCAAATCTCAAAC-3'.

\section{Statistical analysis}

Data were analyzed using the STATA13 software (STATACorp, USA). Statistical analysis of AZM MICs in different test media was performed by non-parametric Kruskal-Wallis test with Dunn's correction for multiple comparison using GraphPad Prism Software. A $p$-value of $<0.05$ was considered statistically significant.

\section{Results}

\section{Study population}

Between January 2013 and December 2016, we received respiratory materials from $930 \mathrm{CF}$ patients. P. aeruginosa was identified in 292 patients out of which 49 (=16.8\%) carried MDR P. aeruginosa (Table 1). MDR was defined according to the rules of the German Commission for Hospital Hygiene and Infection Prevention (KRINKO) as combined resistance to piperacillin, piperacillin/tazobactam, ceftazidime, imipenem, meropenem and ciprofloxacin. This definition is also in line with the one of the North American CF registry $[4,5]$. Random isolates from approximately two-thirds $(n=30 / 49)$ of these MDR $P$. aeruginosa positive patients had been cryopreserved in skim milk at $-80^{\circ} \mathrm{C}$. As two isolates were not cultivable and as one sample contained two different MDR isolates, 29 isolates from 28 patients were finally included in the present study. All patients were classified as chronic $P$.
Table 1 Basic data on microbiological findings within the study population

\begin{tabular}{|c|c|c|}
\hline & CF patients & \\
\hline & $n$ & \\
\hline Total & 930 & \\
\hline P. aeruginosa pos. & 292 & \\
\hline \multirow[t]{3}{*}{ P. aeruginosa MDR pos. ${ }^{a}$} & 49 ( $=16.8 \%$ of $P$. aeruginosa pos.) & \\
\hline & MDR P. aeruginosa, ${ }^{b}$ & \\
\hline & $n$ & $\%$ \\
\hline \multicolumn{3}{|l|}{$\mathrm{Age}^{\mathrm{c}}$} \\
\hline $0-10$ & 3 & 11 \\
\hline $11-20$ & 2 & 7 \\
\hline $21-30$ & 10 & 36 \\
\hline $31-40$ & 7 & 25 \\
\hline $41-50$ & 2 & 7 \\
\hline$>51$ & 4 & 14 \\
\hline \multicolumn{3}{|l|}{ Resistance to ${ }^{d}$} \\
\hline Colistin & 5 & 17 \\
\hline Fosfomycin & 20 & 69 \\
\hline Aztreonam & 29 & 100 \\
\hline Gentamicin & 27 & 93 \\
\hline Tobramycin & 18 & 62 \\
\hline Amikacin & 25 & 86 \\
\hline \multicolumn{3}{|l|}{ Carriage status ${ }^{\mathrm{e}}$} \\
\hline Intermittent & 1 & 4 \\
\hline Chronic & 27 & 96 \\
\hline
\end{tabular}

a defined as combined resistance to piperacillin/tazobactame, ceftazidime, imipenem, meropenem and ciprofloxacin

b $N=29$ isolates from $N=28$ patients, i.e. one patient had two morphologically distinct isolates

c median age 27.5 years (range 7 to 70 years); $n=28$ patients

d categorized as resistant (i.v. application) if MIC interpreted as intermediate or resistant

e chronic: $>50 \%$ P. aeruginosa positive samples within 12 months; intermittent: $<50 \%$ P. aeruginosa positive samples within 12 months; negative: $>1$ year $P$. aeruginosa negative

aeruginosa carriers with the exception of one patient with an intermittent carriage status [19]. Most patients in the study cohort were aged between 21 and 40 years. The youngest patient who was tested positive for MDR $P$. aeruginosa was 7 years old. Co-resistance to other antibiotics in MDR $P$. aeruginosa was common: all isolates were resistant to aztreonam, $69 \%$ to fosfomycin and resistance to aminoglycosides ranged from $62 \%$ (tobramycin) to $93 \%$ (gentamicin). Non-susceptibility to colistin was observed in $17 \%$ of isolates (Table 1 ). For tobramycin and colistin, antimicrobial susceptibility testing was interpreted for systemic administration as neither EUCAST nor CLSI (Clinical \& Laboratory Standards Institute) provide breakpoint values for local application of these antibiotics via inhalation. 


\section{Determination of azithromycin MIC by BMD in MDR $P$. aeruginosa using different test media}

Previous studies suggested that the medium used for BMD critically influences the in vitro susceptibility of $P$. aeruginosa towards azithromycin [11, 12]. Yet it remains unclear if azithromycin exerts direct antimicrobial effects also in MDR $P$. aeruginosa isolates and in $\mathrm{CF}$ adapted test medium. We therefore determined azithromycin MICs in (i) MDR P. aeruginosa CF clinical isolates using (ii) different media for BMD including CAMHB (medium commonly used for BMD), RPMI 1640 (eukaryotic cell culture medium used by $[11,12]$ ) and SCFM (medium mimicking CF airway milieu). Unlike previously described [11], P. aeruginosa reference strain PAO1 failed to grow in RPMI 1640 alone but required the presence of FCS (Fig. 1a). Yet, in line with the data of Buyck et al. [11], azithromycin MICs against PAO1 were $1 \mu \mathrm{g} / \mathrm{ml}$ when measured in RPMI supplemented with $30 \%$ FCS and ranged between 128 and $256 \mu \mathrm{g} / \mathrm{ml}$ in CA-MHB in three independent experiments (Fig. 1b). Surprisingly, in SCFM, azithromycin MIC of PAO1 was reproducibly determined with $\geq 1024 \mu \mathrm{g} / \mathrm{ml}$ and was thus
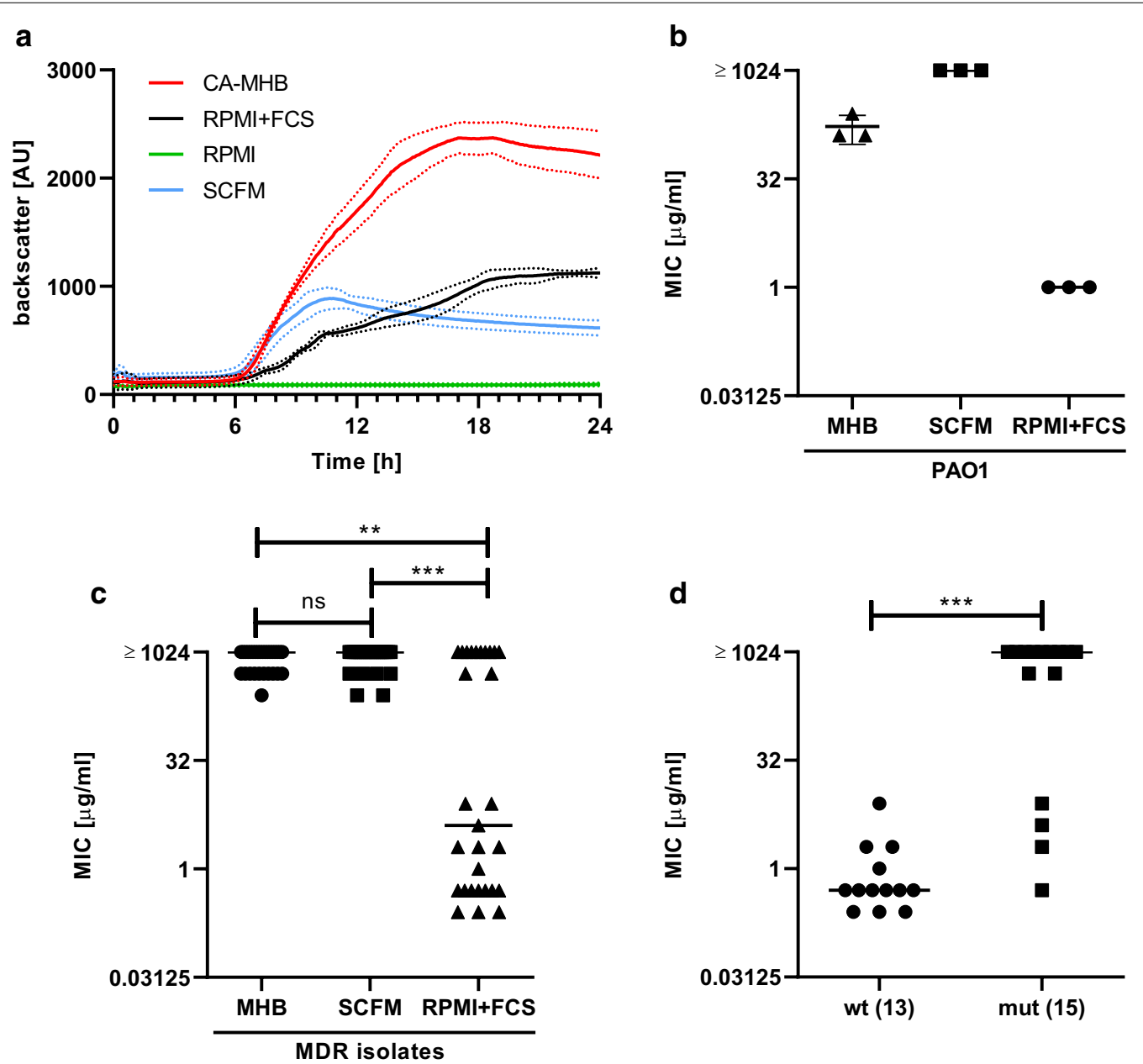

Fig. 1 Evaluation of azithromycin MICs against P. aeruginosa strain PAO1 and MDR P. aeruginosa CF isolates in different media. a P. aeruginosa strain PAO1 was inoculated in the indicated media and increase in bacterial growth was continuously evaluated over $24 \mathrm{~h}$ by measuring backscattered light intensity using Cell Growth Quantifier system. CA-MHB: cation-adjusted Mueller Hinton Broth; RPMI: Roswell Park Memorial Institute 1640 medium; FCS: fetal calf serum; SCFM: synthetic cystic fibrosis sputum medium. Data indicate mean (solid lines) +/ - SD (dotted lines) from three independent experiments. b, c MICs of azithromycin were determined by broth microdilution in P. aeruginosa strain PAO1 (b) and in multidrug resistant (MDR) clinical P. aeruginosa isolates derived from cystic fibrosis patients (c) using CA-MHB, SCFM and RPMI 1640 supplemented with $30 \% \mathrm{FCS}$ as test medium. Bars indicate mean $+/-$ SD from three independent experiments (b) or median values (c). For MDR isolates, $n=29$ for CA-MHB and RPMI/FCS and $n=27$ for SCFM (Two isolates failed to grow in SCFM and were therefore excluded from analysis). Statistical analysis was performed using a Kruskal-Wallis test and Dunn's test for multiple comparison. $\left.\left(^{* *}\right) p<0.01 ;{ }^{* * *}\right) p<0.001$; ns: not significant (d) MIC distribution in RPMI/FCS for strains that showed either wildtype (wt) or mutant (mut) sequence in 23S rRNA (pos. 2045, 2046 and 2598) 
even slightly higher than in CA-MHB (Fig. 1b). Of note, in MDR P. aeruginosa clinical isolates derived from CF patients, two distinct populations became evident when azithromycin MICs were assessed in RPMI/FCS: In 17/29 MDR isolates (59\%), MIC ranged between 0.25 and $8 \mu \mathrm{g} /$ $\mathrm{ml}$ whereas $12 / 29$ MDR isolates (41\%) had a MIC of $\geq 512 \mu \mathrm{g} / \mathrm{ml}$ (Fig. 1c). However, MICs were $\geq 256 \mu \mathrm{g} / \mathrm{ml}$ for all MDR isolates when measured either in CF adapted medium SCFM or conventional CA-MHB (Fig. 1c).

To study whether the mechanism of resistance against azithromycin might affect the differing susceptibility in RPMI/FCS, mutations at three positions within $23 \mathrm{~S}$ rRNA (2045, 2046 and 2598 in Pseudomonas aeruginosa PAO1 corresponding to position 2058, 2059 and 2611 in the $23 \mathrm{~S}$ subunit of Escherichia coli) known to be associated with azithromycin resistance $[12,20]$ were analyzed. We found mutations at one of the three positions in $23 \mathrm{~S}$ rRNA in $53.6 \%$ of the isolates ( 15 out of 28 isolates that could be sequenced) whereas $46.4 \%(N=13)$ showed wildtype sequence at those positions. Four types of mutations were detected: A2045G, A2046C, A2046G, A2598T. Of note, when analyzing the MICs in RPMI/FCS for wildype vs. mutant strains, it became obvious that 11 from 15 mutant strains had MICs $\geq 512 \mu \mathrm{g} / \mathrm{ml}$, whereas for wildtype strains all but one had MIC $<8 \mu \mathrm{g} / \mathrm{ml}$.

\section{Discussion}

Several clinical studies have validated the beneficial effects of long-term treatment with azithromycin in CF patients chronically infected with Pseudomonas aeruginosa ( $P$. aeruginosa) and its usage has progressively entered clinical guidelines [5-9]. The efficiency of azithromycin has been attributed to its anti-inflammatory and anti-virulence properties including e.g. inhibition of motility, quorum sensing and protease activity $[9,10,21,22]$. Although $P$. aeruginosa is considered naturally resistant to macrolides, in vitro susceptibility was previously demonstrated upon testing in alternative media including eukaryotic cell medium RPMI 1640 (supplemented or not with FCS) or serum-supplemented CA-MHB, suggesting that macrolides might additionally exert direct antimicrobial activity on $P$. aeruginosa $[10$, 11]. The differences observed in phenotypic susceptibility to azithromycin depending on the test medium have been ascribed to increased outer-membrane permeability and decreased expression of efflux pumps in the presence of RPMI 1640 or serum, leading to enhanced azithromycin accumulation inside the bacteria [11]. The authors therefore proposed that azithromycin MIC testing of $P$. aeruginosa CF isolates in RPMI 1640 could routinely be included in microbiological diagnostics [11].

Extending previous findings, we demonstrate here that in 17 out of 29 (59\%) MDR P. aeruginosa CF isolates,
MIC values were low when tested in RPMI supplemented with FCS, ranging from $0.25-8 \mu \mathrm{g} / \mathrm{ml}$. In contrast, in vitro resistance with high MICs to azithromycin even in RPMI/FCS as found in 12 out of 29 MDR isolates in the present study could be explained by mutations in the 23S rRNA which are frequently detected in CF isolates. Indeed, Mustafa et al. observed mutations in domain $\mathrm{V}$ of $23 \mathrm{~S}$ rRNA in $43 \%$ of CF $P$. aeruginosa isolates while mutations were absent in 48 tested strains derived from patients suffering from hospital acquired pneumonia [12]. Thus, testing in RPMI/FCS might be an option to identify $P$. aeruginosa resistance caused by $23 \mathrm{~S}$ rRNA mutation.

However, although RPMI and CA-MHB supplemented with FCS have been suggested to more closely resemble the eukaryotic environment and therefore to constitute the better test medium, these media do not necessarily reflect the particular milieu in the airways of CF patients. It was suggested that the physiological situation of $\mathrm{CF}$ airways might be better mimicked by SCFM which imitates the specific nutritional composition and ion concentrations of CF sputum and is therefore a less rich medium compared to RPMI [13]. We therefore evaluated susceptibility of MDR $P$. aeruginosa in this medium. Of note, azithromycin MICs were consistently $\geq 256 \mu \mathrm{g} / \mathrm{ml}$ in SCFM in all $P$. aeruginosa clinical isolates as well as in reference strain PAO1, arguing against a direct antimicrobial effect of azithromycin in the airways of CF patients. Macrolides are protonated in acidic environments going along with reduced activity. SCFM was used with a $\mathrm{pH}$ of 6.8 , which might interfere with activity, yet, a slightly acidic $\mathrm{pH}$ in airways of CF patients is well documented in the literature [13]. As a conclusion, our data therefore do not support routine azithromycin MIC assessment in CF clinical isolates using RPMI/FCS, as proposed previously [12]. This study shows that for CF isolates and macrolides in vitro testing is associated with a high level of uncertainty. SCFM, sputum adapted medium, might be more appropriate for antimicrobial susceptibility testing than conventional broth. This notion is also supported by a recent publication of Diaz Iglesias et al who investigated antibiotic susceptibility, biofilm formation and metabolic activity using different media [23].

Our results do not substantiate a direct antimicrobial effect of azithromycin on $P$. aeruginosa when tested in SCFM, a medium that represents the CF environment. Even though we could not detect an antibacterial effect of azithromycin on $P$. aeruginosa we could replicate the occurrence of a resistance mechanism, specifically the mutation of the 23S rRNA gene, in MDR isolates of CF patients. This might be interpreted as a hint that azithromycin has an effect on $P$. aeruginosa in vivo other than blocking bacterial growth. Indeed it has 
been demonstrated that azithromycin causes a modulation of protein expression instead of a complete block which would be needed for an antibacterial effect. This effect seems to be pronounced for genes involved in quorum sensing [22, 24, 25]. Since this effect seems to take place at concentration far below the measured MIC and the quorum sensing system is involved in biofilm formation, virulence and immune modulation $[26,27]$ it is plausible that this drives the development of azithromycin resistance in $P$. aeruginosa in vivo. Since azithromycin does not seem to be effective in CF patients uninfected with $P$. aeruginosa [28] this indicates that azithromycin has indeed an effect on $P$. aeruginosa in vivo. Therefore it would be interesting to study the effect of azithromycin in patients with azithromycin resistant strains compared to patients with non-resistant strains. In this context testing for azithromycin resistance using RPMI might be a useful tool.

In conclusion our data do not support the implementation of azithromycin MIC assessment of $P$. aeruginosa $\mathrm{CF}$ isolates in routine microbiological diagnostics as suggested previously [12]. The results warrant further assessment of the in vivo efficacy of azithromycin in the subgroup of MDR P. aeruginosa infected CF patients in prospective clinical trial.

\begin{abstract}
Abbreviations
CF: Cystic fibrosis; P. aeruginosa: Pseudomonas aeruginosa; CFU: Colony forming units; SCFM: Synthetic Cystic Fibrosis Sputum Medium; CA-MBH: Cation adjusted Mueller Hinton Broth; RPMI: Roswell Park Memorial Institute; FCS: Fetal calf serum; BMD: Broth microdilution; MIC: Minimal inhibitory concentration; MDR: Multi-drug resistance; LIS: Laboratory information system; CGQ: Cell Growth Quantifier.
\end{abstract}

\section{Acknowledgements}

We thank Suzan Leccese and Selina Hassel for excellent technical support.

\section{Authors' contributions}

MS and TE concepted and supervised the entire study. BK, MW and TE performed experiments. MW, DN, SB, BY, AHD and TE analyzed the data. AHD provided resources and critically discussed the results. TE wrote the initial draft of the manuscript. All authors read and approved the final manuscript.

\section{Funding}

Open Access funding enabled and organized by Projekt DEAL. The authors received no specific grant from any funding agency.

\section{Availability of data and materials}

The datasets used and/or analysed during the current study are available from the corresponding author on reasonable request.

\section{Ethics approval and consent to participate}

The surveillance of multi-resistant organisms is performed in concordance to the German Infection Protection Act. The local ethics advisory board of the Heidelberg University Hospital was consulted prior to study begin for conformity with the current regulations (S-474/2018).

\section{Consent for publication}

Not applicable.

\section{Competing interests}

The authors declare that they have no competing interests.

\section{Author details}

${ }^{1}$ Department of Infectious Diseases, Medical Microbiology and Hygiene, Heidelberg University Hospital, 69120 Heidelberg, Germany. ${ }^{2}$ Laboratory Enders and Partners, 70193 Stuttgart, Germany. ${ }^{3}$ Institute of Medical Microbiology and Hygiene, Medical Faculty, Technische Universität Dresden, Fetscherstraße 74, 01307 Dresden, Germany. ${ }^{4}$ Present Address: SLK-Kliniken Heilbronn, 74080 Heilbronn, Germany.

Received: 21 July 2020 Accepted: 20 November 2020

Published online: 09 December 2020

\section{References}

1. Mall MA, Hartl D. CFTR: cystic fibrosis and beyond. Eur Respir J. 2014:44(4):1042-54.

2. Lund-Palau H, Turnbull AR, Bush A, Bardin E, Cameron L, Soren O, et al. Pseudomonas aeruginosa infection in cystic fibrosis: pathophysiological mechanisms and therapeutic approaches. Expert Rev Respir Med. 2016;10(6):685-97.

3. Mayer-Hamblett N, Kloster M, Rosenfeld M, Gibson RL, Retsch-Bogart GZ, Emerson J, et al. Impact of sustained eradication of new Pseudomonas aeruginosa infection on long-term outcomes in cystic fibrosis. Clin Infect Dis. 2015;61(5):707-15.

4. Report CFFPRAD. https://www.cff.org/Research/Researcher-Resources/ Patient-Registry/2017-Patient-Registry-Annual-Data-Report.pdf Accessed 22 May 2019

5. Stefani S, Campana S, Cariani L, Carnovale V, Colombo C, Lleo MM, et al. Relevance of multidrug-resistant Pseudomonas aeruginosa infections in cystic fibrosis. Int J Med Microbiol. 2017;307(6):353-62.

6. Chalmers JD. Macrolide resistance in Pseudomonas aeruginosa: implications for practice. Eur Respir J. 2017;49:1700689. https://doi. org/10.1183/13993003.00689-2017.

7. Mogayzel PJ Jr, Naureckas ET, Robinson KA, Mueller G, Hadjiliadis D, Hoag JB, et al. Cystic fibrosis pulmonary guidelines. Chronic medications for maintenance of lung health. Am J Respir Crit Care Med. 2013;187(7):680-9.

8. Principi N, Blasi F, Esposito S. Azithromycin use in patients with cystic fibrosis. Eur J Clin Microbiol Infect Dis. 2015;34(6):1071-9.

9. Saiman L, Marshall BC, Mayer-Hamblett N, Burns JL, Quittner AL, Cibene DA, et al. Azithromycin in patients with cystic fibrosis chronically infected with Pseudomonas aeruginosa: a randomized controlled trial. JAMA. 2003;290(13):1749-56.

10. Imperi F, Leoni L, Visca P. Antivirulence activity of azithromycin in Pseudomonas aeruginosa. Front Microbiol. 2014;5:178.

11. Buyck JM, Plesiat P, Traore H, Vanderbist F, Tulkens PM, Van Bambeke F. Increased susceptibility of Pseudomonas aeruginosa to macrolides and ketolides in eukaryotic cell culture media and biological fluids due to decreased expression of oprM and increased outer-membrane permeability. Clin Infect Dis. 2012;55(4):534-42.

12. Mustafa M-H, Khandekar S, Tunney MM, Elborn JS, Kahl BC, Denis O, et al. Acquired resistance to macrolides in Pseudomonas aeruginosa from cystic fibrosis patients. Eur Respir J. 2017:49:1601847. https://doi. org/10.1183/13993003.01847-2016.

13. Palmer KL, Aye LM, Whiteley M. Nutritional cues control Pseudomonas aeruginosa multicellular behavior in cystic fibrosis sputum. J Bacteriol. 2007;189(22):8079-87.

14. Hogardt MH, Susanne; Balke, Beate; Kahl, Barbara C.; Schmoldt, Sabine; Leitritz, Lorenz; Jäger, Gundula; Kappler, Matthias; Suerbaum, Sebastian; Heesemann, Jürgen Atemwegsinfektionen bei Mukoviszidose. Qualitätsstandards in der mikrobiologisch-infektiologischen Diagnostik 2006;:MiQ24.

15. Matuschek E, Ahman J, Webster C, Kahlmeter G. Antimicrobial susceptibility testing of colistin - evaluation of seven commercial MIC products against standard broth microdilution for Escherichia coli, Klebsiella pneumoniae, Pseudomonas aeruginosa, and Acinetobacter spp. Clin Microbiol Infect. 2018;24(8):865-70. 
16. Balouiri M, Sadiki M, Ibnsouda SK. Methods for in vitro evaluating antimicrobial activity: a review. J Pharm Anal. 2016;6(2):71-9.

17. Wiegand I, Hilpert $K$, Hancock RE. Agar and broth dilution methods to determine the minimal inhibitory concentration (MIC) of antimicrobial substances. Nat Protoc. 2008;3(2):163-75.

18. Oberhardt MA, Puchalka J, Fryer KE, Martins dos Santos VA, Papin JA. Genome-scale metabolic network analysis of the opportunistic pathogen Pseudomonas aeruginosa PAO1. J Bacteriol. 2008;190(8):2790-803.

19. Pressler T, Bohmova C, Conway S, Dumcius S, Hjelte L, Hoiby N, et al. Chronic Pseudomonas aeruginosa infection definition: EuroCareCF working group report. J Cyst Fibros. 2011;10(Suppl 2):S75-8.

20. Marvig RL, Sondergaard MS, Damkiaer S, Hoiby N, Johansen HK, Molin $\mathrm{S}$, et al. Mutations in $23 \mathrm{~S}$ rRNA confer resistance against azithromycin in Pseudomonas aeruginosa. Antimicrob Agents Chemother. 2012;56(8):4519-21.

21. Castellani C, Duff AJA, Bell SC, Heijerman HGM, Munck A, Ratjen F, et al. ECFS best practice guidelines: the 2018 revision. J Cyst Fibros. 2018;17(2):153-78.

22. Molinari G, Guzman CA, Pesce A, Schito GC. Inhibition of Pseudomonas aeruginosa virulence factors by subinhibitory concentrations of azithromycin and other macrolide antibiotics. J Antimicrob Chemother. 1993;31(5):681-8.

23. Diaz Iglesias Y, Wilms T, Vanbever R, Van Bambeke F. Activity of antibiotics against Staphylococcus aureus in an in vitro model of biofilms in the context of cystic fibrosis: influence of the culture medium. Antimicrob Agents Chemother. 2019;63(7):e00602-19.

24. Swatton JE, Davenport PW, Maunders EA, Griffin JL, Lilley KS, Welch M. Impact of azithromycin on the quorum sensing-controlled proteome of Pseudomonas aeruginosa. PLoS One. 2016;11(1):e0147698.

25. Godeke J, Pustelny C, Haussler S. Recycling of peptidyl-tRNAs by peptidyl-tRNA hydrolase counteracts azithromycin-mediated effects on Pseudomonas aeruginosa. Antimicrob Agents Chemother. 2013:57(4):1617-24.

26. Scoffone VC, Trespidi G, Chiarelli LR, Barbieri G, Buroni S. Quorum sensing as antivirulence target in cystic fibrosis pathogens. Int J Mol Sci. 2019:20:1838.

27. Kariminik A, Baseri-Salehi M, Kheirkhah B. Pseudomonas aeruginosa quorum sensing modulates immune responses: an updated review article. Immunol Lett. 2017;190:1-6.

28. Saiman L, Anstead M, Mayer-Hamblett N, Lands LC, Kloster M, HocevarTrnka J, et al. Effect of azithromycin on pulmonary function in patients with cystic fibrosis uninfected with Pseudomonas aeruginosa: a randomized controlled trial. JAMA. 2010;303(17):1707-15.

\section{Publisher's Note}

Springer Nature remains neutral with regard to jurisdictional claims in published maps and institutional affiliations.
Ready to submit your research? Choose BMC and benefit from:

- fast, convenient online submission

- thorough peer review by experienced researchers in your field

- rapid publication on acceptance

- support for research data, including large and complex data types

- gold Open Access which fosters wider collaboration and increased citations

- maximum visibility for your research: over $100 \mathrm{M}$ website views per year

At BMC, research is always in progress.

Learn more biomedcentral.com/submissions 